\title{
Delay of Broadband Signals Using Slow Light in Stimulated Brillouin Scattering With Phase-Modulated Pump
}

\author{
Lilin Yi, Li Zhan, Weisheng $\mathrm{Hu}$, and Yuxing Xia
}

\begin{abstract}
Slow light due to stimulated Brillouin scattering in fibers is a promising technique to realize all-optical data storage and synchronization. For delaying high-speed data, we demonstrate an approach to solve the problem of narrowband Brillouin gain by phase-modulating the Brillouin pump. The bit rate of the phase-modulating signal determines the Brillouin gain bandwidth. A $1.25-\mathrm{Gb} / \mathrm{s}$ nonreturn-to-zero pseudorandom binary sequence data is delayed by 520 ps with a phase-modulated pump operating at $2.5-\mathrm{Gb} / \mathrm{s}$ bit rate. We have also observed the pattern-dependent delay and distortion of the data caused by the filtering characteristics of Brillouin amplification, and we have suppressed the distortion by detuning the carrier of the signal away from the Brillouin gain peak.
\end{abstract}

Index Terms-Delay, phase modulation, slow light, stimulated Brillouin scattering (SBS).

\section{INTRODUCTION}

$\mathbf{S}$ LOWING down the group velocity of light pulses by manipulating dispersion is required for an all-optical information storage. The bit-level delay time can be used for synchronization of optical pulses. Slow-light techniques based on fiber nonlinearities, such as stimulated Brillouin scattering (SBS) [1] [3], stimulated Raman scattering [4], and parametric process [5], [6] attract much interest because they are compatible with fiber-optic communication systems and can work at room temperature. Particularly, SBS-based slow light in fiber looks most promising since the signal can work at any wavelength. However, the bit rate of the delayed signal is limited to tens of megabits per second because of the narrowband characteristic of the Brillouin amplification. To increase the Brillouin gain bandwidth to support the delay of higher speed data, a simple pump-spectral broadening technique was used [2]-[4], where the pump bandwidth is broadened by direct-modulation of the pump laser diode (LD) using a pseudorandom binary sequence (PRBS) data [2] and a Gaussian noise source [3].

Recently, we used an external phase modulator (PM) as another choice to broaden the pump spectrum, and demonstrated the delay of return-to-zero PRBS data with $1-\mathrm{Gb} / \mathrm{s}$ bit rate [7]. Compared to the direct modulation, the external modulation has high reliability and the spectral shape is only determined by the bit sequences of the modulated data. Even a flat-top Brillouin

Manuscript received October 30, 2006; revised February 4, 2007.

The authors are with the State Key Laboratory of Advanced Optical Communication Systems and Networks, Shanghai Jiao Tong University, Shanghai 200240, China (e-mail: lizhan@sjtu.edu.cn).

Color versions of one or more of the figures in this letter are available online at http://ieeexplore.ieee.org.

Digital Object Identifier 10.1109/LPT.2007.894350

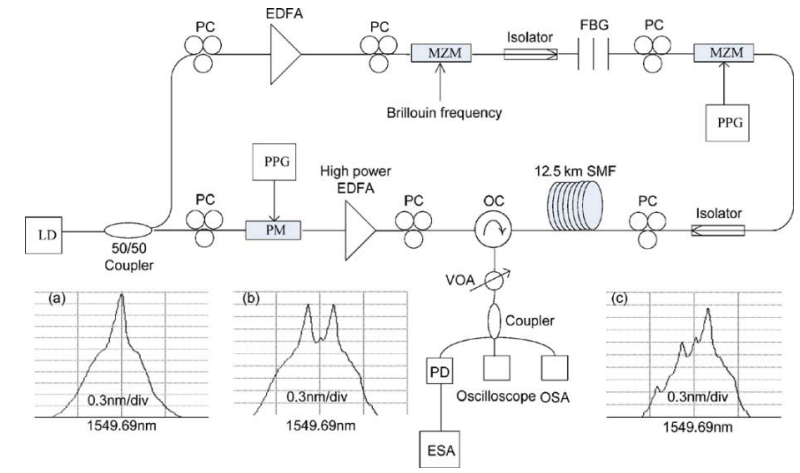

Fig. 1. Experimental setup.

gain spectrum can be achieved by appropriately setting the data sequences [8]. In this letter, we further study the delay performance of the frequently used nonreturn-to-zero (NRZ) data in the wideband Brillouin amplifier with phase-modulated pump, and we observed the data pattern dependent delay and distortion. The pattern dependency of the distortion could be suppressed by detuning the signal carrier away from the Brillouin gain peak. In our demonstration, a 1.25-Gb/s NRZ PRBS data is delayed by $520 \mathrm{ps}$ with phase-modulation of the pump at a bit rate of $2.5 \mathrm{~Gb} / \mathrm{s}$. The delay for higher speed data can be realized using higher speed phase-modulation of the pump.

\section{EXPERIMENT}

The experimental setup is shown in Fig. 1. The output of an LD operating at $1549.69 \mathrm{~nm}$ is divided into two parts by a 50/50 optical coupler for the Brillouin pump and the signal, respectively. In the lower path, the light is modulated by a PM with a maximum modulation-rate of $2.5 \mathrm{GHz}$. PRBS data $\left(2^{23}-1\right)$ from a pulse pattern generator modulate the phase of the light to broaden the optical spectrum. The broadened light is then boosted by a high power erbium-doped fiber amplifier (EDFA) and launched into a $12.5-\mathrm{km}$ single-mode fiber (SMF) by an optical circulator (OC) to serve as the Brillouin pump for the slow-light delay line. The measured Brillouin frequency shift of the fiber is $\sim 10.96 \mathrm{GHz}$. In the upper path, the light is preamplified by another EDFA and then modulated by a Mach-Zehnder modulator (MZM) at the Brillouin frequency of the SMF using the double-sideband suppressed-carrier modulation [9]. A fiber Bragg grating (FBG) with a central wavelength of $1549.60 \mathrm{~nm}$ and a 3-dB bandwidth of $10 \mathrm{GHz}$ reflects the lower sideband of the light. The spectra of the original light, the two sidebands, and the upper sideband of the light are insets (a), (b), and (c) 

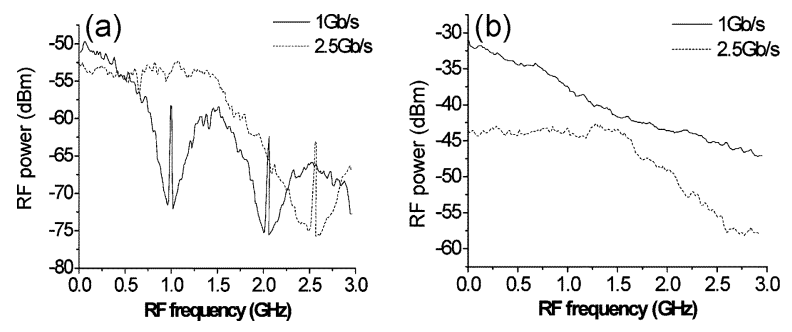

Fig. 2. (a) Pump spectra and (b) Brillouin gain bandwidth measurements taken using an ESA at different bit rates of the pump modulation.

in Fig. 1, respectively. The upper sideband of the light is intensity-modulated using another MZM and then sent to the SMF through an optical isolator to serve as the Brillouin signal. After being amplified and delayed in the SMF, the signal exits from the OC and measurements are performed. A variable optical attenuator is used to control the signal power before the measurements. We use an optical spectrum analyzer, an oscilloscope, a photodetecor, and an electrical spectrum analyzer (ESA) to measure the optical spectra, the signal waveforms, and the electrical spectra, respectively.

\section{RESULTS AND DISCUSSION}

The Brillouin gain bandwidth is determined by the convolution of the pump spectrum and the Brillouin intrinsic gain profile [2]. When the pump is phase-modulated using PRBS data, the pump spectrum is greatly broadened, and the spectral width is controlled by the bit rate of the modulating signal. The broadened pump spectra at different bit rates of the phase modulation are shown in Fig. 2(a) by using the coherent homodyne measurement technique and subsequently sent to an ESA. With a $2.5-\mathrm{Gb} / \mathrm{s}$ bit rate of the modulating signal, the 3 -dB bandwidth of the pump spectrum is $\sim 1.6 \mathrm{GHz}$. Fig. 2(b) shows the baseband response of the Brillouin amplifier at different bit rates of the pump modulation. When the pump is modulated at $2.5 \mathrm{~Gb} / \mathrm{s}$, the $3-\mathrm{dB}$ bandwidth of the Brillouin gain is about $1.6 \mathrm{GHz}$. It is well known that, in all the slow-light schemes based on the narrowband gain, the delay time is approximately proportional to the gain value and inversely proportional to the gain bandwidth, thus the pump-spectral broadening technique reduces the delay time. However, in practical applications, the delay-bandwidth-product, namely the fractional delay of the signal is of important consideration, as it represents the storage capacity of slow-light systems. In the following experiments, we fixed the bit rate of the pump modulation at $2.5 \mathrm{~Gb} / \mathrm{s}$.

To verify the Brillouin gain-bandwidth broadening effect, we show eye diagrams of a $500-\mathrm{Mb} / \mathrm{s}\left(2^{18}-1\right)$ signal in Fig. 3. Fig. 3(a) is the back-to-back eye diagram of the signal. If the pump is not modulated, the signal is totally distorted because of the narrowband Brillouin gain, as shown in Fig. 3(b). When the driving voltage of the PM is not equal to $V_{\pi}(\sim 4 \mathrm{~V})$, the residual carrier of the pump will generate a narrow Brillouin gain bandwidth, which degrades the wideband signal. Only when the driving voltage is equal to or close to $V_{\pi}$ does the carrier component disappear and the broadened pump spectrum generate a wideband Brillouin gain bandwidth, which amplifies the signal without incurring large distortion, as shown in Fig. 3(c).

We then measured the time delay and the pulse distortion with different bit rates of the signal. In Fig. 4, we show that the
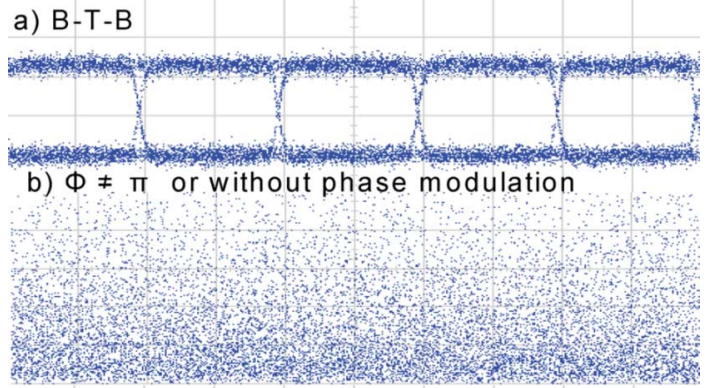

c) $\Phi=\pi$

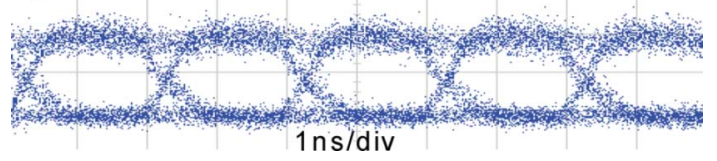

Fig. 3. Eye diagrams of a signal at a $500-\mathrm{Mb} / \mathrm{s}$ rate: (a) back-toback, (b) without phase-modulation of the pump, (c) with a $2.5-\mathrm{Gb} / \mathrm{s}$ phase-modulation of the pump.

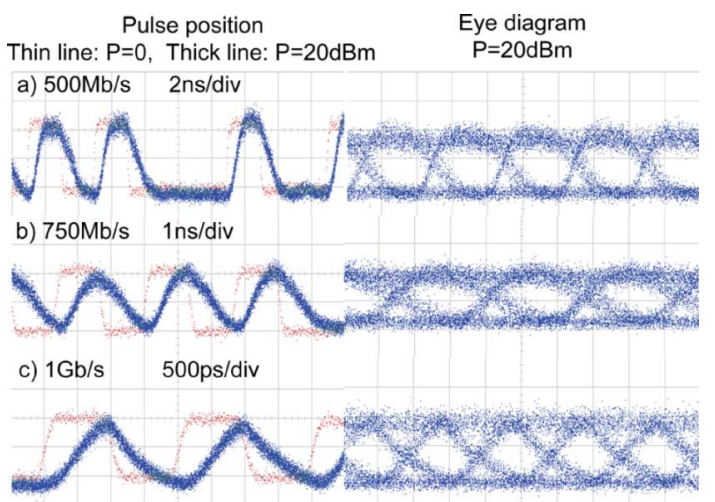

Fig. 4. Signal pulse waveforms and eye diagrams at different bit rates of the signal with a $2.5-\mathrm{Gb} / \mathrm{s}$ phase-modulation of the pump: (a) $500 \mathrm{Mb} / \mathrm{s}$, (b) $750 \mathrm{Mb} / \mathrm{s}$, (c) $1 \mathrm{~Gb} / \mathrm{s}$.

pulse waveforms (left) and the eye diagrams (right) at $500 \mathrm{Mb} / \mathrm{s}$, $750 \mathrm{Mb} / \mathrm{s}$, and $1 \mathrm{~Gb} / \mathrm{s}$ with a word length of $2^{18}-1$, respectively. At $500-\mathrm{Mb} / \mathrm{s}$ rate, when the pump power is $20 \mathrm{dBm}$ which results in a $15-\mathrm{dB}$ gain, the signal pulses experience $800-\mathrm{ps}$ delay, and a clear eye diagram is obtained. Note that we use the temporal center of the amplified pulses to define the pulse position, as the Brillouin amplification is always accompanied with the pulse distortion. With increase in the signal bit rate, the delay-bandwidth-product of the pulses is clearly improved at the same pump power of $20 \mathrm{dBm}$. The $1-\mathrm{Gb} / \mathrm{s}$ signal is delayed by about a half bit. Due to the $1.6-\mathrm{GHz}$ Brillouin gain bandwidth, the delayed 1-Gb/s signal experiences large distortion. The narrow Brillouin gain bandwidth induces the strong dispersion, so it leads to pulse broadening, which is the main cause of signal distortion. The chromatic dispersion of the SMF also broadens the pulses, but that is negligible compared to the strong dispersion of slow light at low bit rate.

We also observed the pattern dependence of the pulse delay and distortion with a $1.25-\mathrm{Gb} / \mathrm{s}$ data and $23-\mathrm{dBm}$ pump power, as shown in Fig. 5. A single " 1 " pulse and consecutive " 1 " pulses experience different delays and distortion, as shown in Fig. 5(a) and (b). The pattern-dependent distortion of the signal results from the Brillouin filtering characteristics. The frequency components of long consecutive " 1 " pulses are 


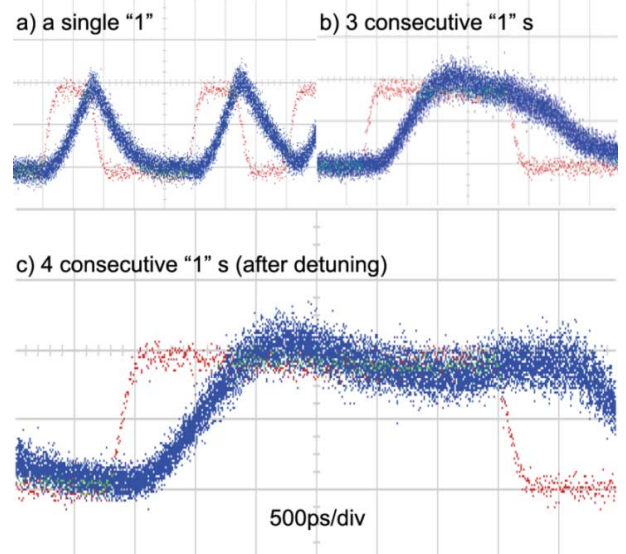

Fig. 5. Pattern dependence of the pulse delay and distortion with a $1.25-\mathrm{Gb} / \mathrm{s}$ data and 23-dBm pump power. (a) Single "1", (b) three consecutive "1"s, (c) four consecutive "1"s after detuning the carrier.

closer to the carrier which lies at the peak of the Brillouin gain profile, so they experience larger gain and delay than a single "1" pulse, whose frequency components are symmetrically distributed at both sides of the carrier and around the low gain region of the Brillouin gain spectrum. In Fig. 5(b), the delay of three consecutive "1" pulses is as large as $\sim 1$ ns, and the distortion also is more severe than that of a single " 1 " pulse. The pattern dependence of the pulse delay and distortion are another important factor of the data degradation in SBS-based slow light. In theory detuning the signal carrier away from the slow-light gain peak can reduce the pattern-dependent effect, as the long consecutive "1" pulses and the single " 1 " pulse can achieve similar gain and delay [10]. We have proved experimentally that this theory works for SBS-based slow light. In our experiment, by detuning the modulation frequency of the first MZM from the Brillouin frequency, the delayed pulse waveforms with less distortion can be obtained as shown in Fig. 5(c).

Finally, we measured the signal gain and the delay time versus the pump power at $500-\mathrm{Mb} / \mathrm{s}, 750-\mathrm{Mb} / \mathrm{s}, 1-\mathrm{Gb} / \mathrm{s}$, and $1.25-\mathrm{Gb} / \mathrm{s}$ data rates of the signal, respectively, as shown in Fig. 6. For the same pump power, the signal gain decreases with increase in the bit rate; therefore, the corresponding delay time is reduced. All the delay times are measured for a single "1" pulse. Increasing the signal bit rate will reduce the delay time at the same pump power, but increase the delay-bandwidth-product. At the 23-dBm pump power, which results in a 12-dB gain, the delay time of a $1.25-\mathrm{Gb} / \mathrm{s}$ signal is about $520 \mathrm{ps}$, corresponding to the delay-bandwidth-product of 0.65 . This parameter can be improved with an increase in the signal bit rate if higher speed phase-modulation of the pump is achievable. Note that the fractional delay in most of the slow-light techniques is limited at the bit-level, which is far from realizing optical buffers, but this delay can be used for bit-synchronization in the future all-optical networks.

\section{CONCLUSION}

We broadened the Brillouin gain bandwidth by phase-modulating the pump, which enables the robust delay
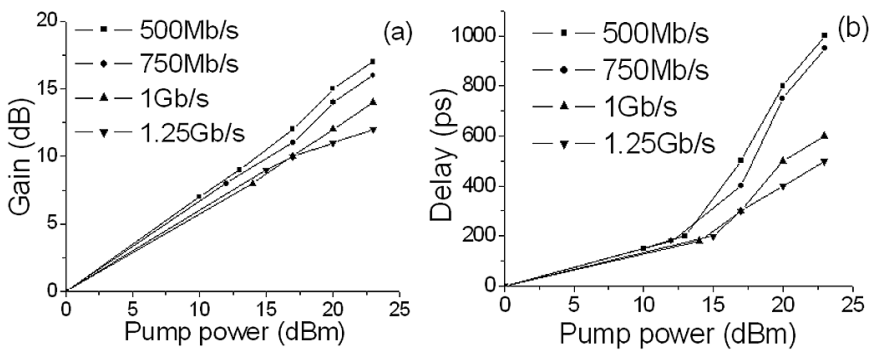

Fig. 6. (a) Gain versus the pump power at different data rates; (b) the delay time versus the pump power at different data rates.

operation of the wideband signal in SBS-based slow-light scheme. In our particular demonstration, a $1.25-\mathrm{Gb} / \mathrm{s} \mathrm{NRZ}$ PRBS data is delayed by about 520 ps with a $2.5-\mathrm{Gb} / \mathrm{s}$ phase modulation of the pump, corresponding to a 0.65 delay-bandwidth-product. We also studied the pattern-dependent pulse delay and distortion, meanwhile, suppressing the pattern-dependent effects by detuning the carrier away from the Brillouin gain peak. Such a wideband slow-light delay-line would be a promising candidate for bit-synchronization in the future all-optical network.

\section{ACKNOWLEDGMENT}

The authors are profoundly thankful to Prof. Y. Su for his valuable discussion during the process of the research.

\section{REFERENCES}

[1] K. Y. Song, M. G. Herráez, and L. Thévenaz, "Observation of pulse delaying and advancement in optical fibers using stimulated Brillouin scattering," Opt. Express, vol. 13, no. 1, pp. 82-88, Jan. 2005.

[2] M. G. Herraez, K. Y. Song, and L. Thevenaz, "Arbitrary-bandwidth Brillouin slow light in optical fibers," Opt. Express, vol. 14, no. 4, pp. 1395-1400, Feb. 2006.

[3] Z. Zhu, A. M. C. Dawes, D. J. Gauthier, L. Zhang, and A. E. Willner, "12-GHz-bandwidth SBS slow light in optical fibers," presented the Proc. OFC, Anaheim, CA, 2006, Paper PDP1.

[4] J. E. Sharping, Y. Okawachi, and A. L. Gaeta, "Wide bandwidth slow light using a Raman fiber amplifier," Opt. Express, vol. 12, no. 16, pp. 6092-6098, Aug. 2005.

[5] D. Dahan and G. Eisenstein, "Tunable all optical delay via slow and fast light propagation in a Raman assisted fiber optical parametric amplifier: A route to all optical buffering," Opt. Express, vol. 13, no. 16, pp. 6234-6249, Aug. 2005.

[6] L. Yi, W. Hu, Y. Su, M. Gao, and L. Leng, "Design and system demonstration of a tunable slow-light delay line based on fiber parametric process," IEEE Photon. Technol. Lett., vol. 18, no. 24, pp. 2575-2577, Dec. 15, 2006.

[7] L. Yi, L. Zhan, Y. Su, W. Hu, L. Leng, Y. Song, H. Shen, and Y. Xia, "Delay of RZ PRBS data based on wideband SBS by phase-modulating the Brillouin pump," presented at the Proc. ECOC, Cannes, France, 2006, Paper We3.P.30.

[8] T. Tanemura, Y. Takushima, and K. Kikuchi, "Narrowband optical filter, with a variable transmission spectrum, using stimulated Brillouin scattering in optical fiber," Opt. Lett., vol. 27, no. 17, Sep. 2002.

[9] M. Nikles, L. Thevenaz, and P. A. Robert, "Brilloui gain spectrum characterization in single-mode optical fibers," J. Lightw. Technol., vol. 15, no. 10 , pp. 1842-1851, Oct. 1997.

[10] L. Zhang, T. Luo, W. Zhang, C. Yu, Y. Wang, and A. E. Willner, "Optimizing operating conditions to reduce data pattern dependence induced by slow light elements," presented at the Proc. OFC, Anaheim, CA, 2006, Paper OFP7. 